Algebraic 85 Geometric Topology

Volume 3 (2003) 993-1004

Published: 5 October 2003

ATG

\title{
On three-periodic trajectories of multi-dimensional dual billiards
}

\author{
SERge TABAChNiKov
}

\begin{abstract}
We consider the dual billiard map with respect to a smooth strictly convex closed hypersurface in linear $2 m$-dimensional symplectic space and prove that it has at least $2 m$ distinct 3 -periodic orbits.
\end{abstract}

AMS Classification 37J45, 70H12

Keywords Dual billiards, symplectic relation, periodic orbits, Morse and Lusternik-Schnirelman theory

\section{Introduction and formulation of results}

The dual billiard map is an outer counterpart of the usual billiard ball map. In the plane, the dual (or outer) billiard map $T$ is defined as follows. Let $M \subset \mathbf{R}^{2}$ be a smooth closed strictly convex curve, and let $z$ be a point in its exterior. There are two tangent lines to $M$ through $z$; choose one of them (say, the right one from the view-point of $z$ ) and define $T(z)$ to be the reflection of $z$ in the point of tangency. J. Moser put forward the study of dual billiards in $[14,15]$, and by now, there exists a substantial literature on the subject: see $[1,4,5,10,11,13,18,19,20,21,22,23,24,25]$. Exotic as they may seem, dual billiards are close relatives of the usual, inner ones; on the sphere, the two systems are isomorphic via the projective duality.

Most of the research done on dual billiards concerned the plane case. However dual billiards can be defined in multi-dimensional setting as well, [19, 20, 21]. This definition is not widely known and is not immediately obvious, so we reproduce it here. One wants to replace the curve $M$ by a hypersurface in Euclidean space, and then the problem is that one has too many tangent lines to $M$ through a given point. This difficulty is resolved as follows. One takes the linear symplectic space $\left(\mathbf{R}^{2 m}, \omega\right)$ where $\omega=\sum d x_{i} \wedge d y_{i}, i=1, \ldots, m$, as the ambient space. Let $M^{2 m-1} \subset \mathbf{R}^{2 m}$ be a smooth hypersurface and $q \in M$. The restriction of $\omega$ to the tangent hyperplane $T_{q} M$ has a 1-dimensional kernel 
$\xi(q)$, called the characteristic line. An orientation of $M$ induces an orientation of $\xi(q)$. This direction is used as the tangent line at $q$ to define the dual billiard map.

Definition 1 Two points $z_{1}, z_{2} \in \mathbf{R}^{2 m}$ are in the dual billiard relation with respect to a smooth hypersurface $M$ if the mid-point $q=\left(z_{1}+z_{2}\right) / 2$ lies on $M$ and the line $\left(z_{1} z_{2}\right)$ coincides with the characteristic line $\xi(q)$.

In the plane (and for a convex dual billiard curve), this is the definition of the dual billiard map, given above. One can prove that the dual billiard relation is a symplectic relation. One can also prove that if $M$ is a smooth closed strictly convex hypersurface then, for every point $z_{1}$ outside of $M$, there exist exactly two points $z_{2}$ such that $z_{1}$ and $z_{2}$ are in the dual billiard relation with respect to $M$. Moreover, for one choice of the point $z_{2}$, the orientation of the ray $z_{1} z_{2}$ coincides with that of $\xi(q)$, and for another the orientation is opposite (just as in the plane). Thus one defines the dual billiard map $T$, a symplectomorphism of the exterior $M$; see $[19,20,21]$.

The dual billiard map may be considered as a discretization of the characteristic flow on $M$; the relation between the dual billiard map and the characteristic flow is the same as between the usual billiard map and the geodesic flow on the boundary of the billiard table, see discussions in [19, 20] and [21], section 4.5.

An important problem concerning a dynamical system is to estimate the number of its periodic trajectories. There is a natural action of the dihedral group $D_{n}$ on the set of $n$-periodic trajectories $\left(z_{1}, \ldots, z_{n}\right), T z_{i}=z_{i+1}$ for $i=1, \ldots, n$ : one can cyclically permute the points $z_{i}$ or change their cyclic order to the opposite. When counting periodic trajectories, we always count the number of $D_{n}$-orbits.

For strictly convex smooth plane dual billiards the situation is the same as for the usual billiards inside a strictly convex closed smooth plane curve: for every period $n \geq 2$ and a every rotation number $1 \leq r \leq n / 2$, coprime with $n$, there exist at least two distinct $n$-periodic dual billiard trajectories with the rotation number $r$. For the usual billiards, this is Birkhoff's theorem [3], and the result extends to area-preserving twist maps of an annulus, of which the plane dual billiard map is a particular case.

Concerning multi-dimensional dual billiards, the only known result so far was the following theorem, see [19, 20,21]: for every smooth closed strictly convex hypersurface in linear symplectic space and every odd prime $n$, the dual billiard map has an $n$-periodic trajectory. This is a rather weak estimate that can be considerably improved. 
A comparison with the usual billiards is in order. Historically the first estimate below of the number of periodic trajectories in multi-dimensional convex billiards is due to I. Babenko [2] where the 3-dimensional case is considered. This case is technically hardest, and [2] contained an error. Satisfactory lower bounds were recently obtained for a generic smooth closed strictly convex billiard hypersurface $M^{m-1} \subset \mathbf{R}^{m}$ by M. Farber and the present author in $[8,9]$ : for an odd $n \geq 3$, the number of distinct $n$-periodic billiard trajectories inside $M^{m-1}$ is not less than $(n-1)(m-1)$. Related results, in particular, on the number of non-closed billiard trajectories going from one point to another are contained in $[7,12]$.

Our goal in this paper is to estimate below the number of 3-periodic trajectories of a strictly convex smooth dual billiard. Period 3 is smallest possible for the dual billiard map, and as such, it is analogous to period 2 for the usual billiard. Two-periodic billiard trajectories are also called diameters or double normals: these are chords of a hypersurface $M^{m-1} \subset \mathbf{R}^{m}$ that are perpendicular to $M$ at both end points. If $M^{m-1}$ is smooth and strictly convex then there are at least $m$ such chords; this is one of the earliest applications of the MorseLusternik-Schnirelman theory. An extension to immersed submanifolds, not necessarily of codimension 1 , is contained in [26], and further generalizations and improvements in $[16,17]$ and $[6]$, where the case of generalized 3-periodic trajectories is considered.

Our main result is as follows.

Theorem 1 For every smooth closed strictly convex hypersurface $M^{2 m-1}$ in $\mathbf{R}^{2 m}$ the number of distinct 3-periodic trajectories of the dual billiard map is not less than $2 m$. This lower bound is sharp: for every $m$ there exists a smooth closed strictly convex hypersurface $M^{2 m-1} \subset \mathbf{R}^{2 m}$ for which this number is equal to $2 m$.

It is an interesting problem to extend this result to greater periods, even, as well as odd, that is, to find a dual billiard analog of $[8,9]$, and to include not necessarily convex immersed hypersurfaces, in the spirit of $[6,12,16,17]$.

\section{Proofs}

Our proof makes use of the critical point theory. The first step is to find a function whose critical points correspond to periodic trajectories. For the usual 
billiards, this function is the perimeter length on inscribed polygons; for dual billiards it is the symplectic area counted with certain multiplicities.

Consider a smooth hypersurface $M^{2 m-1} \subset \mathbf{R}^{2 m}$. Let $n$ be an odd number and $\left(z_{1}, \ldots, z_{n}\right)$ an $n$-tuple of points in $\mathbf{R}^{2 m}$. Set: $q_{i}=\left(z_{i}+z_{i+1}\right) / 2$ where the index $i=1, \ldots, n$ is understood cyclically. The following result is contained in $[19,20,21]$.

Lemma 2.1 The points $\left(z_{1}, \ldots, z_{n}\right)$ form an $n$-periodic orbit of the dual billiard relation with respect to $M$ if and only if the points $q_{1}, \ldots, q_{n}$ lie on $M$ and the $n$-tuple $\left(q_{1}, \ldots, q_{n}\right)$ is a critical point of the function on $M \times \ldots \times M$ given by the formula:

$$
F\left(q_{1}, \ldots, q_{n}\right)=\sum_{1 \leq i<j \leq n}(-1)^{i+j} \omega\left(q_{i}, q_{j}\right) .
$$

Proof By Definition 1, points $z_{k}$ and $z_{k+1}$ are in the dual billiard relation if and only if $q_{k} \in M$ and the vector $z_{k+1}-z_{k}$ is parallel to $\xi\left(q_{k}\right)$.

It is convenient to introduce the complex structure $J$ :

$$
\omega(u, v)=J u \cdot v=-u \cdot J v .
$$

Let $\nu(q)$ be the unit normal vector to $M$ at point $q \in M$. Then the vector $J \nu(q)$ is parallel to the characteristic line $\xi(q)$.

Consider $\bar{q}=\left(q_{1}, \ldots, q_{n}\right) \in M \times \ldots \times M$. Then

$$
d F(\bar{q})=\sum_{1 \leq i<j \leq n}(-1)^{i+j}\left(J q_{i} d q_{j}-J q_{j} d q_{i}\right) .
$$

By the Lagrange multipliers method, the point $\bar{q}$ is critical for $F$ if and only if

$$
d F(\bar{q})=\sum_{k} \lambda_{k} \nu\left(q_{k}\right) d q_{k}
$$

where $\lambda_{k}$ are Lagrange multipliers. Fix $k$, collect the terms in front of $d q_{k}$ in (2) and (3) and equate:

$$
(-1)^{k} \sum_{j=0}^{n-2}(-1)^{j} q_{k+1+j}=-\lambda_{k} J \nu\left(q_{k}\right) .
$$

Since $n$ is odd one can express $z$ in terms of $q$ :

$$
z_{i}=\sum_{j=0}^{n-1}(-1)^{j} q_{i+j}
$$

Algebraic 83 Geometric Topology, Volume 3 (2003) 
and hence

$$
z_{k+1}-z_{k}=2 \sum_{j=0}^{n-2}(-1)^{j} q_{k+1+j} .
$$

Compare (5) with (4) to conclude that $\bar{q}$ is a critical point if and only if $z_{k+1}-z_{k}$ has the characteristic direction at $q_{k}$ for all $k=1, \ldots, n$. This means that the points $z_{k}$ and $z_{k+1}$ are in the dual billiard relation.

Note that the function $F$ does not depend on the choice of the origin. Note also that the dihedral group $D_{n}$ acts on the arguments of $F$ by permutations, and $F$ is an odd function with respect to this action: $F$ is invariant under cyclic permutations and changes sign if the order is reversed. This property distinguishes $F$ from the perimeter length function in the usual billiard set-up which is invariant under $D_{n}$. For $n=3$, the case under consideration here, $F$ is (negative) the symplectic area of a triangle inscribed into the dual billiard hypersurface $M$ :

$$
F\left(q_{1}, q_{2}, q_{3}\right)=\omega\left(q_{2}, q_{1}\right)+\omega\left(q_{3}, q_{2}\right)+\omega\left(q_{1}, q_{2}\right) .
$$

Assume now that $M$ is strictly convex so that the dual billiard map $T$ is defined. Most of the critical points of $F\left(q_{1}, \ldots, q_{n}\right)$ do not correspond to $n$-periodic orbits of $T$ but rather to backtracking "fake" orbits $\left(\ldots, z_{i-1}, z_{i}, z_{i+1}, \ldots\right)$ with $z_{i-1}=z_{i+1}$. This backtracking occurs when $q_{i-1}=q_{i}$. Therefore, to estimate the number of $n$-periodic orbits of $T$ one needs to consider the critical points of $F$ on the cyclic configuration space

$$
G(M, n)=\left\{\left(q_{1}, \ldots, q_{n}\right) \in M \times \ldots \times M \mid q_{i-1} \neq q_{i} \text { for } i=1, \ldots, n\right\}
$$

whose topology was studied in [8]. This critical set is acted upon by $D_{n}$, and each $D_{n}$ orbit corresponds to a single $n$-periodic orbit of $T$.

From now on, $n=3$, the dihedral group $D_{3}$ coincides with the symmetric group $S_{3}$, and the function $F$ is skew-symmetric, see (6). In particular, if $q_{i}=q_{j}$ for some $i \neq j$ then $F\left(q_{1}, q_{2}, q_{3}\right)=0$. To prove Theorem 1 we use MorseLusternik-Schnirelman theory. One cannot immediately apply this theory to $G(M, 3)$ because this space is open. A way around this difficulty is as follows.

Choose a sufficiently small generic $\varepsilon>0$ and consider the manifold with boundary

$$
U=\left\{\left(q_{1}, q_{2}, q_{3}\right) \in M \times M \times M \mid F\left(q_{1}, q_{2}, q_{3}\right) \leq-\varepsilon\right\} .
$$

Then $U \subset G(M, 3)$. The group of cyclic permutations $\mathbf{Z}_{3}$ acts on $U$ freely; let $V=U / \mathbf{Z}_{3}$. The function $F$ descends to a function $\bar{F}$ on $V$, therefore 
the number of 3-periodic trajectories of the dual billiard map is not less than the number of critical points of $\bar{F}$. The negative gradient of $\bar{F}$ has the inward direction along the boundary $\partial V$, and the usual estimates of the MorseLusternik-Schnirelman theory apply. In particular, the number of 3-periodic orbits of $T$ is not less than the sum of Betti numbers of $V$ and greater than the cohomological length of this space.

The critical points of $F$ in the set $F\left(q_{1}, q_{2}, q_{3}\right) \geq \varepsilon$ are in one-to-one correspondence with those in $U$, due to skew-symmetry of the function $F$. However one may lose critical points on the zero level set, that is, 3-periodic orbits that lie in an isotropic affine 2-plane. In fact, no such orbits exist, as the next lemma shows.

Lemma 2.2 A non-degenerate triangle with zero symplectic area, inscribed into $M$, is not critical for the symplectic area functional.

Proof Assume that $q_{1}, q_{2}, q_{3}$ is an inscribed triangle with zero symplectic area, corresponding to a 3-periodic orbit of $T$. Then the vector $q_{3}-q_{2}$ is parallel to the characteristic direction $\xi\left(q_{1}\right)$. Since the symplectic area vanishes, $\omega\left(q_{3}-\right.$ $\left.q_{2}, q_{2}-q_{1}\right)=0$, and hence the vector $q_{2}-q_{1}$ lies in the symplectic orthogonal complement to $\xi\left(q_{1}\right)$, that is, the tangent hyperplane $T_{q_{1}} M$. This contradicts strict convexity of $M$.

We identify the homotopy equivalence class of $V$ in two steps. Let $W$ be the set of orthonormal 2-frames $\left(e_{1}, e_{2}\right)$ in $\mathbf{R}^{2 m}$ with $\omega\left(e_{1}, e_{2}\right)>0$. One has a free $\mathbf{Z}_{3}$ action on $W$ by rotating a frame in the plane spanned by $e_{1}$ and $e_{2}$ through $2 \pi / 3$ in the direction from $e_{1}$ to $e_{2}$.

Lemma 2.3 $U$ is $\mathbf{Z}_{3}$-equivariant homotopy equivalent to $W$.

Proof Given a triangle $q_{1} q_{2} q_{3} \in U$, parallel translate it in such a way that its center of mass is at the origin. Then dilate the position vectors of the vertices of the triangle to unit vectors. We now have three pairwise distinct unit vectors $z_{1}, z_{2}, z_{3}$ in $\mathbf{R}^{2 m}$ that form a triangle of positive symplectic area. Let $P$ be the 2-plane of this triangle.

Consider the function

$$
\phi\left(z_{1}, z_{2}, z_{3}\right)=\left|z_{1}-z_{2}\right|+\left|z_{2}-z_{3}\right|+\left|z_{3}-z_{1}\right| .
$$

This is a smooth function on the cyclic configuration space $G\left(S^{1}, 3\right)$ of the unit circle in the plane $P$. The critical points of $\phi$ are 3 -periodic billiard trajectories 
inside the circle, that is, equilateral triangles. The open manifold $G\left(S^{1}, 3\right)$ is homotopically equivalent to the manifold with boundary given by the condition

$$
\left|z_{1}-z_{2}\right|\left|z_{2}-z_{3}\right|\left|z_{3}-z_{1}\right| \geq \delta
$$

where $\delta>0$ is generic and sufficiently small. The gradient of $\phi$ has the inward direction along the boundary and defines a retraction of $G\left(S^{1}, 3\right)$ to the set of inscribed equilateral triangles (see, e.g., [8] for details). An equilateral triangle in the plane $P$ is determined by its first vertex $e_{1}$, and since the restriction of $\omega$ on $P$ does not vanish, the choice of $e_{1}$ uniquely determines $e_{2}$ so that $\omega\left(e_{1}, e_{2}\right)>0$.

The above construction provides a $Z_{3}$-equivariant map from $U$ to $W$; this map gives the desired homotopy equivalence.

Let $\lambda=(-1+\sqrt{-3}) / 2$ be the primitive cube root of 1 . Consider the standard action of $\mathbf{Z}_{3}$ on the sphere $S^{2 m-1} \subset \mathbf{C}^{m}$ by multiplying every coordinate by $\lambda$.

Lemma 2.4 $W$ is $\mathbf{Z}_{3}$-equivariant homotopy equivalent to $S^{2 m-1}$.

Proof We already identified $\mathbf{R}^{2 m}$ with $\mathbf{C}^{m}$ by introducing the operator $J$. We want to show that $W$ is $\mathbf{Z}_{3}$-equivariant homotopy equivalent to the set of orthonormal 2-frames $\left(e_{1}, e_{2}\right)$ with $e_{2}=J e_{1}$.

Consider the function $\psi\left(e_{1}, e_{2}\right)=\omega\left(e_{1}, e_{2}\right)$ on the Stiefel manifold of orthonormal 2 -frames. Note that $\psi$ is $\mathbf{Z}_{3}$-invariant. We claim that the critical points of $\psi$ consist of two critical manifolds, the complex and anticomplex frames $e_{2}= \pm J e_{1}$. Indeed, let $\left(e_{1}, e_{2}\right)$ be a frame and $\left(u_{1}, u_{2}\right)$ its variation. Then

$$
e_{1} \cdot u_{1}=e_{2} \cdot u_{2}=e_{1} \cdot u_{2}+e_{2} \cdot u_{1}=0 .
$$

One has:

$$
d \psi\left(u_{1}, u_{2}\right)=J e_{1} \cdot u_{2}-J e_{2} \cdot u_{1} .
$$

Therefore, by $(7),\left(e_{1}, e_{2}\right)$ is a critical point if and only if the vector $\left(-J e_{2}, J e_{1}\right)$ is a linear combination of the three vectors $\left(e_{1}, 0\right),\left(0, e_{2}\right),\left(e_{2}, e_{1}\right)$. This is possible only when $e_{2}= \pm J e_{1}$.

To complete the proof of the lemma, one chooses a generic $\delta>0$ and replaces $W$ by a manifold with boundary $\omega\left(e_{1}, e_{2}\right) \geq \delta$. Then the gradient of $\psi$ retracts $W$ to the set of complex frames $\left(e_{1}, e_{2}\right)$ with $e_{2}=J e_{1}$, and this is the desired $Z_{3}$-equivariant homotopy equivalence. 
It follows from Lemmas 2.3 and 2.4 that $V$ is homotopy equivalent to the lens space $S^{2 m-1} / \mathbf{Z}_{3}$. The cohomological length of the lens space with coefficients in $\mathbf{Z}_{3}$ is $2 m-1$. It follows from the Lusternik-Schnirelman theory that the function $\bar{F}$ has at least $2 m$ distinct critical points on $V$. This proves the lower bound of Theorem 1 .

We construct a hypersurface in $\mathbf{R}^{2 m}$ for which the number of 3-periodic dual billiard trajectories equals $2 m$ by a small perturbation of the unit sphere $S^{2 m-1} \subset \mathbf{R}^{2 m}$. We describe $M$ in terms of its support function.

The support function of a smooth strictly convex closed hypersurface $M^{2 m-1}$ is the function on the unit sphere $h: S^{2 m-1} \rightarrow \mathbf{R}$ whose value on $z \in S^{2 m-1}$ is the maximum of the linear function $\phi_{z}(x)=x \cdot z$ on $M$. Let $q(z) \in M$ be the point at which the maximum is attained. Then

$$
q(z)=h(z) z+\nabla h(z)
$$

where the position vector $z$ and the gradient $\nabla h(z)$ are considered as vectors in $\mathbf{R}^{2 m}$. The vector $z$ is the outward unit normal vector to $M$ at $q$, and the characteristic direction at $q$ is given by the vector $J z$. A triple of distinct points $z_{1}, z_{2}, z_{3} \in S^{2 m-1}$ corresponds to a 3 -periodic orbit of the dual billiard map with respect to $M$ if and only if there exist real numbers $a_{1}, a_{2}, a_{3}$ such that

$$
q_{i}+a_{i} J z_{i}=q_{i+1}-a_{i+1} J z_{i+1}, \quad i=1,2,3,
$$

where $q_{i}$ are related to $z_{i}$ as in (8).

Lemma 2.5 Let $f$ be a smooth $\mathbf{Z}_{3}$-invariant function on $S^{2 m-1}$ that has $N$ critical $\mathbf{Z}_{3}$-orbits. Consider the hypersurface $M$ with the support function $h=1+\varepsilon f$. Then, for sufficiently small $\varepsilon$, the number of 3-periodic orbits of the respective dual billiard map does not exceed $N$.

Proof Consider first the case when $h=1$, that is, $M$ is the unit sphere. Recall that $\lambda$ denotes the primitive cube root of 1 . Then, for every $z \in S^{2 m-1}$, the triple $z_{i}=\lambda^{i} z$ is a solution of (9) with all $a_{i}=\sqrt{3}$, and every solution of (9) is of this form.

Next let $h=1+\varepsilon f$. We consider the linearizations of (8) and (9) in $\varepsilon$. The number of solutions of the linearized system provides an upper bound on the number of genuine solutions.

We are looking for a perturbed solution in the form

$$
z_{i}=\lambda^{i}\left(z+\varepsilon v_{i}\right), a_{i}=\sqrt{3}+\varepsilon \alpha_{i}, \quad i=1,2,3,
$$


where $z \in S^{2 m-1}$ and $v_{1}, v_{2}, v_{3} \in T_{z} S^{2 m-1}$ (there is redundancy in the choice of vectors $v_{i}$, for example, one may assume that $\left.v_{3}=0\right)$. All computations are made modulo $\varepsilon^{2}$. Denote the common value of $f(z), f(\lambda z)$ and $f\left(\lambda^{2} z\right)$ by $c$. Likewise, one has:

$$
\nabla f(z)=\lambda^{-1} \nabla f(\lambda z)=\lambda^{-2} \nabla f\left(\lambda^{2} z\right),
$$

and we denote this tangent vector at $z$ by $w$.

Substitute (10) to (8) to obtain:

$$
q\left(z_{i}\right)=\lambda^{i}\left[z+\varepsilon\left(v_{i}+c z+w\right)\right], \quad i=1,2,3 .
$$

Next, substitute to (9) and collect terms linear in $\varepsilon$ :

$$
\frac{\left(c+\sqrt{-1} \alpha_{i}\right) z+w+(1+\sqrt{-3}) v_{i}}{\lambda}=\left(c-\sqrt{-1} \alpha_{i+1}\right) z+w+(1-\sqrt{-3}) v_{i+1}
$$

for $i=1,2,3$.

Note that $\lambda(1-\sqrt{-3})=1+\sqrt{-3}$, hence the coefficients of $v_{i}$ and $v_{i+1}$ in (11) are equal. Add the three equations (11) to obtain:

$$
\left(3(1-\lambda) c+(1+\lambda) \sqrt{-1}\left(\alpha_{1}+\alpha_{2}+\alpha_{3}\right)\right) z=3(\lambda-1) w .
$$

Since $w$ is perpendicular to $z$, we conclude that $w=0$. Thus $z$ is a critical point of the function $f$. Note that once such a critical point $z$ is chosen, one finds from (11) that all $\alpha_{i}=\sqrt{3} c$ and then solves the linear system (11) for vectors $v_{i}$.

To summarize, each critical $\mathbf{Z}_{3}$-orbit of the function $f$ contributes a solution to (11), that is, corresponds, in the linear approximation, to a 3-periodic orbit of the dual billiard map.

To prove the second statement of Theorem 1 we construct a $\mathbf{Z}_{3}$-invariant function on $S^{2 m-1}$ with $2 m$ critical $\mathbf{Z}_{3}$-orbits. Let $x_{1}, \ldots, x_{m}, y_{1}, \ldots, y_{m}$ be Euclidean coordinates in $\mathbf{R}^{2 m}$ so that $x_{i}+\sqrt{-1} y_{i}, i=1, \ldots, m$ are complex coordinates in $\mathbf{C}^{m}$.

Lemma 2.6 Let $a_{1}, \ldots, a_{m}$ be distinct reals. Then, for sufficiently small $\varepsilon$, the function

$$
f\left(x_{1}, \ldots, x_{m}, y_{1}, \ldots, y_{m}\right)=\sum_{i=1}^{m} a_{i} \frac{x_{i}^{2}+y_{i}^{2}}{2}+\varepsilon \sum_{i=1}^{m} \frac{x_{i}^{3}-3 x_{i} y_{i}^{2}}{3}
$$

is $\mathbf{Z}_{3}$-invariant on $S^{2 m-1}$ and has $2 m$ critical $\mathbf{Z}_{3}$-orbits. 
Proof The first term in (12) is invariant under multiplication by complex numbers with modulus 1 . The second term consists of $\operatorname{Re}\left(\left(x_{i}+\sqrt{-1} y_{i}\right)^{3}\right)$, hence it is invariant under multiplication by $\lambda$. To find critical points of $f$ on the unit sphere, we use the Lagrange multipliers method:

$$
a_{i} x_{i}+\varepsilon\left(x_{i}^{2}-y_{i}^{2}\right)=\eta x_{i}, \quad a_{i} y_{i}-2 \varepsilon x_{i} y_{i}=\eta y_{i}, \quad i=1, \ldots, m,
$$

where $\eta$ is a Lagrange multiplier. For each $i$, the system (13) has four solutions:

$$
x_{i}=\frac{\eta-a_{i}}{\varepsilon}, y_{i}=0 ; x_{i}=-\frac{\eta-a_{i}}{2 \varepsilon}, y_{i}= \pm \frac{\sqrt{3}\left(\eta-a_{i}\right)}{2 \varepsilon}
$$

and $x_{i}=y_{i}=0$. The solutions (14) form a $\mathbf{Z}_{3}$-orbit for which $x_{i}^{2}+y_{i}^{2}=$ $\left(\eta-a_{i}\right)^{2} / \varepsilon^{2}$.

For $I \subset\{1, \ldots, m\}$ consisting of at least 2 elements, consider the function

$$
g_{I}(\eta)=\sum_{i \in I}\left(\eta-a_{i}\right)^{2}
$$

and let

$$
\delta=\min _{\eta} \min _{|I| \geq 2} g_{I}(\eta) .
$$

Since all $a_{i}$ are distinct, $\delta>0$. Let $\varepsilon$ be so small that $\delta>\varepsilon^{2}$.

We claim that for every solution of the system (13) there exists a unique index $i \in\{1, \ldots, m\}$ for which (14) holds, and $x_{j}=y_{j}=0$ for $j \neq i$. Indeed, assume that (14) occurs for indices in a set $I$ with $|I| \geq 2$. Since the domain of the function is the unit sphere, one has:

$$
1=\sum_{i=1}^{m}\left(x_{i}^{2}+y_{i}^{2}\right)=\sum_{i \in I} \frac{\left(\eta-a_{i}\right)^{2}}{\varepsilon^{2}} \geq \frac{\delta}{\varepsilon^{2}},
$$

contradicting the choice of $\varepsilon$.

Finally, there are $m$ choices of an index $i \in\{1, \ldots, m\}$, and for each choice one has $\left(\eta-a_{i}\right)^{2}=\varepsilon^{2}$. This gives two values of $\eta$ and two respective solutions (14). Thus the function $f$ has $2 m$ critical $\mathbf{Z}_{3}$-orbits.

Acknowledgments I am grateful to M. Farber for numerous stimulating discussions and to D. Handron for explaining his work on billiard trajectories. Part of this work was done at Max-Planck-Institut in Bonn; it is a pleasure to acknowledge the Institute's invariable hospitality. This work was partially supported by BSF and NSF grants. 


\section{References}

[1] E. Amiran, Lazutkin coordinates and invariant curves for outer billiards. J. Math. Phys., 36 (1995), 1232-1241.

[2] I. Babenko, Periodic trajectories of three-dimensional Birkhoff billiards. Math. USSR, Sbornik, 71 (1992), 1-13.

[3] G. Birkhoff, Dynamical systems. Amer. Math. Soc. Coll. Publ., 9, 1927.

[4] Ph. Boyland, Dual billiards, twist maps and impact oscillators. Nonlinearity, 9 (1996), 1411-1438.

[5] F. Dogru, S. Tabachnikov, On polygonal dual billiard in the hyperbolic plane. Reg. Chaotic Dynamics, 8 (2003), 67-82.

[6] F. Duzhin, Lower bounds for the number of closed billiard trajectories of period 2 and 3 in manifolds embedded in Euclidean space. Int. Math. Res. Notes, 2003, no. 8, 425-449.

[7] M. Farber, Topology of billiard problems. I, II. Duke Math. J., 115 (2002), 559-585, 587-621.

[8] M. Farber, S. Tabachnikov, Topology of cyclic configuration spaces and periodic trajectories of multi-dimensional billiards. Topology, 41 (2002), 553-589.

[9] M. Farber, S. Tabachnikov, Periodic trajectories in 3-dimensional convex billiards. Manuscripta Math., 108 (2002), 431-437.

[10] E. Gutkin, A. Katok, Caustics for inner and outer billiards. Comm. Math. Phys., 173 (1995), 101-134.

[11] E. Gutkin, N. Simanyi, Dual polygonal billiards and necklace dynamics. Comm. Math. Phys., 143 (1991), 431-450.

[12] D. Handron, Generalized billiard paths and Morse theory for manifolds with corners. Topology Appl., 126 (2002), 83-118.

[13] R. Kolodziej, The antibilliard outside a polygon. Bull. Pol. Acad. Sci., 37 (1989), 163-168.

[14] J. Moser, Stable and random motions in dynamical systems. Ann. of Math. Stud., 77, 1973.

[15] J. Moser, Is the solar system stable? Math. Intell., 1 (1978), 65-71.

[16] P. Pushkar', Diameters of immersed manifolds and of wave fronts. C. R. Acad. Sci., 326 (1998), 201-205.

[17] P. Pushkar', A generalization of Chekanov's theorem. Diameters of immersed manifolds and wave fronts. Proc. Steklov Inst. Math., 1998, no. 2 (221), 279-295.

[18] A. Shaidenko, F. Vivaldi, Global stability of a class of discontinuous dual billiards. Comm. Math. Phys., 110 (1987), 625-640.

[19] S. Tabachnikov, Outer billiards. Russian Math. Surv., 48 (1993), No. 6, 81109. 
[20] S. Tabachnikov, On the dual billiard problem. Adv. Math., 115 (1995), 221249.

[21] S. Tabachnikov, Billiards. Soc. Math. France, 1995.

[22] S. Tabachnikov, Poncelet's theorem and dual billiards. L'Enseign. Math., 39 (1993), 189-194.

[23] S. Tabachnikov, Commuting dual billiards. Geom. Dedicata, 53 (1994), 57-68.

[24] S. Tabachnikov, Asymptotic dynamics of the dual billiard transformation. J. Stat. Phys., 83 (1996), 27-38

[25] S. Tabachnikov, Dual billiards in the hyperbolic plane. Nonlinearity, 15 (2002), 1051-1072

[26] F. Takens, J. White, Morse theory of double normals of immersions. Indiana Univ. Math. J., 21 (1971/1972), 11-17.

Department of Mathematics

Pennsylvania State University

University Park, PA 16802, USA

Email: tabachni@math.psu.edu

URL: www.math.psu.edu/tabachni

Received: 21 Febuary 2003

Algebraic 83 Geometric Topology, Volume 3 (2003) 